\title{
Design and Comprehensive Analysis of A Noise-Tolerant ZNN Model with Limited-Time Convergence for Time-Dependent Nonlinear Minimization
}

\author{
Lin Xiao, Jianhua Dai, Rongbo Lu, Shuai Li, Jichun Li and Shoujin Wang
}

\begin{abstract}
Zeroing neural network (ZNN) is a powerful tool to address mathematical and optimization problems broadly arisen in science and engineering areas. The convergence and robustness are always co-pursued in ZNN. However, there exists no related work on ZNN for time-dependent nonlinear minimization that achieves simultaneously limited-time convergence and inherently noise suppression. In this paper, for the purpose of satisfying such two requirements, a limited-time robust neural network (LTRNN) is devised and presented to solve time-dependent nonlinear minimization under various external disturbances. Different from previous ZNN model for this problem either with limited-time convergence or with noise suppression, the proposed LTRNN model simultaneously possesses such two characteristics. Besides, rigorous theoretical analyses are given to prove the superior performance of the LTRNN model when adopted to solve timedependent nonlinear minimization under external disturbances. Comparative results also substantiate the effectiveness and advantages of LTRNN via solving a time-dependent nonlinear minimization problem.
\end{abstract}

Index Terms-Zhang neural networks, zeroing neural networks, nonlinear minimization, time-varying, limited-time convergence, robustness.

\section{INTRODUCTION}

$\mathbf{N}$ ONLINEAR minimization is one of the most important branches of optimization for many scientific and engineering applications [1]-[9]. For example, optimal path tracking of robot manipulators was usually formulated as nonlinear minimization problems solving [1], [4], [7], [9]. Other practical applications (e.g., multiagent systems, image processing and restoration) can also be handled by modelling and solving nonlinear minimization problems [3], [8]. A amount of research attention was focused on finding exact

This work is supported in part by the National Natural Science Foundation of China under Grant 61866013, Grant 61503152, Grant 61976089, Grant 61473259 , and Grant 61563017, and in part by the Natural Science Foundation of Hunan Province of China under Grant 2019JJ50478, Grant 18A289, Grant JGY201926, Grant 2016JJ2101, Grant 2018TP1018, and Grant 2018RS3065.

L. Xiao and J. Dai are with the Hunan Provincial Key Laboratory of Intelligent Computing and Language Information Processing, Hunan Normal University, Changsha 410081, China. (e-mail: xiaolin860728@163.com).

R. Lu is with College of Information Science and Engineering, Jishou University, Jishou 416000, China.

$\mathrm{S}$. Li is with Department of Computing, The Hong Kong Polytechnic University, Hong Kong.

J. Li is with the School of Science, Engineering and Design, Teesside University, Middlesbrough TS1 3BX, U.K.

S. Wang is with the department of computing, Macquarie University, NSW 2109, Australia solutions of nonlinear minimization problem, and various numerical algorithms were developed and analyzed in existing literature [10]-[15]. The classical algorithm for nonlinear minimization is Newton iterative method [12]-[14] which converges quadratically under mild conditions. Different improved algorithms based on Newton iterative method were developed to modify the computing efficiency and comprehensive performance [13]-[16].

As we know, numerical algorithms are of serial computing patterns. In dealing with large-scale nonlinear minimization problems, high computational complexity is unavoidable because of their intrinsic shortcomings [17]. Besides, solving time-dependent (or say, time-varying) nonlinear minimization problem is a challenging task because such a problem is varying with time [18]-[21]. We need to compute the exact solution of time-dependent nonlinear minimization at each time, all of which consist of exact time-varying solutions. Since time-dependent nonlinear minimization requires high computation efficiency, most iterative algorithms, which are effective on time-invariant nonlinear minimization, don't work well enough on time-dependent nonlinear minimization [16].

Recently, recurrent neural networks (RNNs) have gained more and more attentions because they have good performance for various practical applications, such as robotics, optimization computation, winner-take-all competition [22][25]. Differing with the numerical algorithms, RNNs are of parallel computing patterns. Therefore, computation efficiency can be greatly enhanced by using RNNs [22]-[25]. RNNs also played an important role in solving nonlinear minimization [16], [26]-[30]. Gradient neural network (GNN) is a typical RNN, which was developed to effectively solve static nonlinear minimization. However, GNN will generate a relatively large lag error when dealing with the time-independent situation. Under such circumstances, a novel continuous-time RNN (called zeroing neural network, ZNN) was devised and analyzed for various time-dependent problems solving; e.g., Sylvester equation [31], matrix inversion [32], [33] quadratic programming [34], and Lyapunov equation [35]. The main advantage of ZNN is able to provide the capability to address the time-independent problems with the exponential convergence [36]-[38]. In [16], [39], [40], ZNN was firstly explored to solve time-dependent and static nonlinear minimizations. The corresponding discrete-time ZNN (DTZNN) model was developed to solve time-dependent nonlinear minimization [39]. 
However, in this work, the independent variable of nonlinear minimization is only one-dimensional. In [40], Jin and Zhang further investigated vector-valued nonlinear minimization that is an extension of scalar-valued nonlinear minimization. In addition, the solution of such a vector-valued nonlinear minimization was applied to manipulator motion generation via using ZNN. Various higher-order DTZNN models were further presented and analyzed to solve the vector-valued nonlinear minimization by using different Taylor-type difference formulas [16], [23]. Compared with previous DTZNN model, higherorder discrete-time ZNN models attained better computational performance [16], [23]. However, in these existing ZNN models for nonlinear minimization, only convergence property was considered while robustness property has not been discussed [16], [39]-[42].

Different from previous research ideas, in this work, we aim to modify the comprehensive property of ZNN models by devising a different formula from a viewpoint of continuoustime systems. As we know, robustness and convergence are two important features, which influence the performance of solving practical time-dependent nonlinear minimizations. In addition, the mentioned $\mathrm{ZNN}$ models for this problem require infinite time for convergence under the ideal conditions. That is to say, external disturbances or noises are not considered in the existing $\mathrm{ZNN}$ models for time-dependent nonlinear minimization. In this work, based on ZNN, a new limitedtime robust neural network (LTRNN) is devised and presented to solve time-dependent nonlinear minimization in front of external disturbances. Different from previous $\mathrm{ZNN}$ models for this problem [16], [39], [40], LTRNN can simultaneously achieve limited-time convergence and suppression of external disturbances. Besides, rigorous theoretical analyses are given to prove the superior performance of the LTRNN model when adopted to solve time-dependent nonlinear minimization under external disturbances. Comparative results also substantiate the effectiveness and advantages of LTRNN via solving a timedependent nonlinear minimization problem. The following is a summary of the major contributions of this paper.

1) The first contribution is the design and analysis of a limited-time robust neural network (LTRNN) model for time-dependent nonlinear minimization. The proposed LTRNN model can simultaneously achieve limited-time convergence and inherently noise suppression.

2) The second contribution is the performance analysis of the proposed LTRNN model. The excellent performance of the proposed model is theoretically guaranteed by theoretical calculation on the upper bound of limitedtime convergence and discussion of the noise suppression property of LTRNN.

3) The third contribution is the demonstration of the computational power of the proposed LRTNN model through applications for specific time-dependent nonlinear minimization. Performance improvements are obviously observed over existing ZNN models.

\section{Problem Description And ZNN Model}

This part of the paper provides problem description of timedependent nonlinear minimization, which is obviously different from static nonlinear minimization. Next, two different ZNN models from previous work are presented for solving time-dependent nonlinear minimization for comparison purpose.

\section{A. Problem Description}

we are concerned with the following time-dependent nonlinear minimization problem solving [16], [39], [40]:

$$
\min _{\mathbf{x}(t) \in R^{n}} g(\mathbf{x}(t), t), \quad \forall t \in[0, \infty)
$$

where $t$ represents time and $g(\cdot, \cdot): R^{n} \times R \rightarrow R$ represents a smooth nonlinear objective function. The goal of the current work is to compute unknown $\mathbf{x}(t) \in R^{n}$ at each time instant $t$ under external disturbances so that the value of nonlinear objective function at each time instant achieves the minimum within finite time, which constitutes the dynamic minimum motion trajectory of $g(\mathbf{x}(t), t)$ for all $t$. In order to assure that equation (1) has only one optimal solution, we consider the situation that $g(\cdot, \cdot)$ is a convex function at each time instant in this work.

From previous studies on nonlinear minimization [16], [39], [40], we conclude that the optimal solution of equation (1) can be obtained via zeroing the partial derivative of nonlinear objective function $g(\mathbf{x}(t), t)$ with respective to $\mathbf{x}(t)$ at time instant. In this case, we introduce a new function $\mathbf{z}(\mathbf{x}(t), t)$ such that it satisfies the following condition:

$$
\mathbf{z}(\mathbf{x}(t), t)=\frac{\partial g(\mathbf{x}(t), t)}{\partial \mathbf{x}(t)}=\mathbf{0} \in R^{n}
$$

where $\frac{\partial g(\mathbf{x}(t), t)}{\partial \mathbf{x}(t)}=\left[\frac{\partial g(\mathbf{x}(t), t)}{\partial x_{1}}, \frac{\partial g(\mathbf{x}(t), t)}{\partial x_{2}}, \cdots, \frac{\partial g(\mathbf{x}(t), t)}{\partial x_{n}}\right]^{\mathrm{T}}=\mathbf{0}$. Thus, the optimal solution of Eq. (1) is equivalent to the solution of the above system of nonlinear equations. In other words, via the above transform, we only need to solve Eq. (2) to equivalently find the optimal solution of time-dependent nonlinear minimization (1).

\section{B. Zeroing Neural Network}

For completeness of this work, ZNN was developed for such time-dependent nonlinear minimization [31], [32], [39], [40], [43]. How it has been designed is simply illustrated via equivalently solving the above nonlinear equation system (2).

In the first place, based on the transformation of timedependent nonlinear minimization (2), we are capable of defining a monitor error function as below:

$$
\mathbf{e}(t)=\left[\frac{\partial g(\mathbf{x}(t), t)}{\partial x_{1}}, \frac{\partial g(\mathbf{x}(t), t)}{\partial x_{2}}, \cdots, \frac{\partial g(\mathbf{x}(t), t)}{\partial x_{n}}\right]^{\mathrm{T}},
$$

where $\mathbf{e}(t)$ is a vector-valued error function, and the timedependent behavior of each element itself can be monitored. If $\mathbf{e}(t)=\mathbf{0}$ is checked, the corresponding solution is what we want.

Next, the following first-order nonlinear dynamic system is designed to make sure $\mathbf{e}(t)$ converge to zero:

$$
\dot{\mathbf{e}}(t)+\varpi \Phi(\mathbf{e}(t))=\mathbf{0},
$$


where $\varpi>0$ is a design parameter exploited to adjust the above dynamic system to converge to the equilibrium point, and $\Phi(\cdot)$ stands for a nonlinear activation function array with each element denoted by $\phi(\cdot)$.

Substituting (3) into (4), we are further capable of deriving the following expression:

$$
\left\{\begin{array}{c}
\frac{\partial^{2} g(\mathbf{x}(t), t)}{\partial x_{1} \partial t}+\varpi \phi\left(\frac{\partial g(\mathbf{x}(t), t)}{\partial x_{1}}\right)=0 \\
\frac{\partial^{2} g(\mathbf{x}(t), t)}{\partial x_{2} \partial t}+\varpi \phi\left(\frac{\partial g(\mathbf{x}(t), t)}{\partial x_{2}}\right)=0 \\
\vdots \\
\frac{\partial^{2} g(\mathbf{x}(t), t)}{\partial x_{n} \partial t}+\varpi \phi\left(\frac{\partial g(\mathbf{x}(t), t)}{\partial x_{n}}\right)=0 .
\end{array}\right.
$$

In addition, $\forall i=1,2, \cdots, n$, we have

$$
\begin{aligned}
\frac{\partial^{2} g}{\partial x_{i} \partial t}= & \frac{\partial^{2} g}{\partial x_{i} \partial x_{1}} \dot{x}_{1}(t)+\frac{\partial^{2} g}{\partial x_{i} \partial x_{2}} \dot{x}_{2}(t)+ \\
& \cdots+\frac{\partial^{2} g}{\partial x_{i} \partial x_{n}} \dot{x}_{n}(t)+\frac{\partial^{2} g}{\partial x_{i} \partial t}
\end{aligned}
$$

where $g$ is used to denote $g(\mathbf{x}(t), t)$ for presentation convenience.

After combining the above derivation results and considering $\partial g(\mathbf{x}(t), t) / \partial \mathbf{x}(t)=\mathbf{z}(\mathbf{x}(t), t)$, we obtain the following zeroing neural network (ZNN) for calculating time-dependent nonlinear minimization (1) and the resultant nonlinear equation system (2):

$$
\mathbf{Q}(\mathbf{x}(t), t) \dot{\mathbf{x}}(t)=-\varpi \Phi(\mathbf{z}(\mathbf{x}(t), t))-\frac{\partial \mathbf{z}(\mathbf{x}(t), t)}{\partial t},
$$

where coefficient matrix $\mathbf{Q}(\mathbf{x}(t), t)$ and vector $\frac{\partial \mathbf{z}(\mathbf{x}(t), t)}{\partial t}$ are defined as

$$
\begin{gathered}
\mathbf{Q}(\mathbf{x}(t), t)=\left[\begin{array}{cccc}
\frac{\partial^{2} g}{\partial x_{1} \partial x_{1}} & \frac{\partial^{2} g}{\partial x_{1} \partial x_{2}} & \cdots & \frac{\partial^{2} g}{\partial x_{1} \partial x_{n}} \\
\frac{\partial^{2} g}{\partial x_{2} \partial x_{1}} & \frac{\partial^{2} g}{\partial x_{2} \partial x_{2}} & \cdots & \frac{\partial^{2} g}{\partial x_{2} \partial x_{n}} \\
\vdots & \vdots & \ddots & \vdots \\
\frac{\partial^{2} g}{\partial x_{n} \partial x_{1}} & \frac{\partial^{2} g}{\partial x_{n} \partial x_{2}} & \cdots & \frac{\partial^{2} g}{\partial x_{n} \partial x_{n}}
\end{array}\right], \\
\frac{\partial \mathbf{z}(\mathbf{x}(t), t)}{\partial t}=\left[\begin{array}{llll}
\frac{\partial^{2} g}{\partial x_{1} \partial t} & \frac{\partial^{2} g}{\partial x_{2} \partial t} & \cdots & \frac{\partial^{2} g}{\partial x_{n} \partial t}
\end{array}\right]^{\mathrm{T}} .
\end{gathered}
$$

Besides, it has been proved that such a ZNN model (6) is capable of converging to the optimal solution of nonlinear minimization as well as nonlinear equation system (2).

\section{Robust Zeroing Neural Network}

Note that the above ZNN model (6) for time-dependent nonlinear minimization does not consider the impact of external disturbances, and may lose efficacy when external noises are injected. For modifying the robustness of ZNN model (6), in 2015 , an inherent noise-tolerance design formula for ZNN was presented in [41], [42], which is repeated as below for easy reading:

$$
\dot{\mathbf{e}}(t)+\gamma_{1} \mathbf{e}(t)+\gamma_{2} \int_{0}^{t} \mathbf{e}(\tau) \mathrm{d} \tau=\mathbf{0}
$$

where $\gamma_{1}>0$ and $\gamma_{2}>0$ stand for two different scaling factors. It has been proved that design formula (7) possesses the inherent noise-tolerance ability, even in front of dynamic noises. However, in design formula (7), nonlinear activation function $\Phi(\cdot)$ is deleted, which makes (7) only achieve the exponential convergence (i.e., infinite-time convergence) [44][46], although the inherent noise tolerance is considered for the design of $\mathrm{ZNN}$.

Based on the inherent noise-tolerance design formula (7), we are capable of gaining the robust zeroing neural network (RZNN) for time-dependent nonlinear minimization (1) via substituting (3) into it:

$$
\mathbf{Q} \dot{\mathbf{x}}=-\gamma_{1} \mathbf{z}-\gamma_{2} \int_{0}^{t} \mathbf{z d} \tau-\frac{\partial \mathbf{z}}{\partial t},
$$

where independent variable $t$ is omitted for presentation convenience; and coefficient matrix $\mathbf{Q}$ and vector $\frac{\partial \mathbf{z}}{\partial t}$ are defined as the same ones of ZNN model (6). In addition, it has been proved that RZNN model (8) is capable of solving timedependent nonlinear minimization (1) under various additive noises [41], [42]. However, due to elimination of nonlinear activation function $\Phi(\cdot)$, such a RZNN model (8) is not able to reach limited-time convergence [44]-[46].

\section{Limited-Time Robust NeURAL Network}

Considering the limitations of the above two ZNN models, in this part, the LTRNN model is devised and studied to solve time-dependent nonlinear minimization (1) as well as the equivalent nonlinear equation system (2). Before that, a new second-order nonlinear dynamic system is developed and analyzed in details, which is used to establish the LTRNN model. Compared to ZNN model (6) and RZNN model (8) for time-dependent nonlinear minimization (1), the proposed LTRNN model simultaneously possesses the limited-time convergence and inherent noise tolerance.

\section{A. Second-Order Nonlinear Formula}

The aforementioned first-order nonlinear formula (4) purely considers the convergence property, which may confine its real-time applications when external disturbances exist; while the nonlinear formula (7) only considers the inherent noisetolerance property, which may confine its online computing applications. That is to say, such two nonlinear formulas have either limited-time convergence or noise suppression property [41], [42], [44]-[46]. In order to overcome this limitation, a new second-order nonlinear system is devised to realize limited-time convergence and noise tolerance. The specific expression is formulated as the following second-order nonlinear system:

$$
\dot{\mathbf{e}}(t)+\gamma_{1} \Phi(\mathbf{e}(t))+\gamma_{2} \Phi\left(\mathbf{e}(t)+\gamma_{1} \int_{0}^{t} \Phi(\mathbf{e}(\tau)) \mathrm{d} \tau\right)=0
$$

where $\gamma_{1}>0, \gamma_{2}>0$, and $\Phi(\cdot)$ are defined the same as before. In addition, the following theoretical results are given to demonstrate the advantages of the proposed second-order nonlinear system (9).

Theorem 1: The second-order nonlinear system (9) is globally stable as long as $\Phi(\cdot)$ is a monotonic increasing odd function. 
Proof: Note that (9) is a vector-valued function. We first consider the $j$ th subsystem of (9), which is described as $(\forall j \in$ $1,2, \cdots, n)$ :

$$
\dot{e}_{j}(t)+\gamma_{1} \phi\left(e_{j}(t)\right)+\gamma_{2} \phi\left(e_{j}(t)+\gamma_{1} \int_{0}^{t} \phi\left(e_{j}(\tau)\right) \mathrm{d} \tau\right)=0
$$

where $\phi(\cdot)$ is the element of $\Phi(\cdot)$. Then, we are capable of defining an auxiliary variable $u_{j}(t)$, and its expression is described as

$$
u_{j}(t)=e_{j}(t)+\gamma_{1} \int_{0}^{t} \phi\left(e_{j}(\tau)\right) \mathrm{d} \tau
$$

Taking a derivative of (11) with respect to time $t$, we have

$$
\dot{u}_{j}(t)=\dot{e}_{j}(t)+\gamma_{1} \phi\left(e_{j}(t)\right) \text {. }
$$

Combining (10), (11) and (12) yields to the following fact:

$$
\dot{u}_{j}(t)+\gamma_{2} \phi\left(u_{j}(t)\right)=0
$$

which is exactly the $j$ th subsystem of (4). Based on the previous conclusion [31], [32], [39], [40], [43], we know that such a subsystem (13) is capable of converging to its equilibrium point, even within finite time provided that $\phi(\cdot)$ is selected appropriately.

Next, let us consider the following Lyapunov function candidate $s_{j}(t)$ for the $j$ th subsystem (10):

$$
s_{j}(t)=\frac{1}{2} \zeta e_{j}^{2}(t)+\frac{1}{2} u_{j}^{2}(t)
$$

where $\zeta>0$ and $s_{0}=s_{j}(0)=\zeta e_{j}^{2}(0) / 2+u_{j}^{2}(0) / 2$ with $e_{j}(0)$ and $u_{j}(0)$ known. Its time derivative is derived as

$$
\begin{aligned}
\frac{\mathrm{d} s_{j}(t)}{\mathrm{d} t}= & \zeta e_{j}(t) \dot{e}_{j}(t)+u_{j}(t) \dot{u}_{j}(t) \\
= & \zeta e_{j}(t)\left[\dot{u}_{j}(t)-\gamma_{1} \phi\left(e_{j}(t)\right)\right]-\gamma_{2} u_{j}(t) \phi\left(u_{j}(t)\right) \\
= & -\zeta \gamma_{2} e_{j}(t) \phi\left(u_{j}(t)\right)-\zeta \gamma_{1} e_{j}(t) \phi\left(e_{j}(t)\right) \\
& -\gamma_{2} u_{j}(t) \phi\left(u_{j}(t)\right)
\end{aligned}
$$

Since $\phi(\cdot)$ is a monotonic increasing odd function, we can apply the mean-value theorem to further simplify the above expression. Thus, we have

$$
\phi\left(u_{j}(t)\right)-\phi(0)=\left.\left(u_{j}(t)-0\right) \frac{\partial \phi\left(u_{j}(\vartheta)\right)}{\partial u_{j}}\right|_{u_{j}(\vartheta) \in R} .
$$

In addition, in a similar way, we can also conclude $\phi(0)=0$ and $\partial \phi\left(u_{j}(t)\right) / \partial u_{j}>0$. Thus, from (16), the following result can be further derived as

$$
\left|\phi\left(u_{j}(t)\right)\right| \leq a_{0}\left|u_{j}(t)\right|,
$$

where $a_{0}=\left.\max \left\{\partial \phi\left(u_{j}(t)\right) / \partial u_{j}\right\}\right|_{u_{j}(t) \in R}>0$. Furthermore, we have

$$
\begin{aligned}
\left|e_{j}(t) \phi\left(u_{j}(t)\right)\right| & \leq\left|e_{j}(t)\right| \cdot\left|\phi\left(u_{j}(t)\right)\right| \\
& \leq a_{0}\left|e_{j}(t)\right| \cdot\left|u_{j}(t)\right| .
\end{aligned}
$$

Let us substitute (17) back into (15), and the following fact is gained:

$$
\begin{aligned}
\frac{\mathrm{d} s_{j}(t)}{\mathrm{d} t}= & -\zeta \gamma_{2} e_{j}(t) \phi\left(u_{j}(t)\right)-\zeta \gamma_{1} e_{j}(t) \phi\left(e_{j}(t)\right) \\
& -\gamma_{2} u_{j}(t) \phi\left(u_{j}(t)\right) \\
\leq & \zeta \gamma_{2}\left|e_{j}(t) \phi\left(u_{j}(t)\right)\right|-\zeta \gamma_{1} e_{j}(t) \phi\left(e_{j}(t)\right) \\
& -\gamma_{2} u_{j}(t) \phi\left(u_{j}(t)\right) \\
\leq & \zeta \gamma_{2} a_{0}\left|e_{j}(t)\right| \cdot\left|u_{j}(t)\right|-\zeta \gamma_{1} a_{1} e_{j}^{2}(t)-\gamma_{2} a_{2} u_{j}^{2}(t) \\
= & -\zeta\left(\sqrt{\gamma_{1} a_{1}}\left|e_{j}\right|-\frac{\gamma_{2} a_{0}}{2 \sqrt{\gamma_{1} a_{1}}}\left|u_{j}(t)\right|\right)^{2} \\
& -\zeta\left(\frac{\gamma_{2} a_{2}}{\zeta}-\frac{\gamma_{2}^{2} a_{0}^{2}}{4 \gamma_{1} a_{1}}\right) u_{j}^{2}(t)
\end{aligned}
$$

where coefficients $a_{1}=\left.\min \left\{\partial \phi\left(e_{j}(t)\right) / \partial e_{j}\right\} \quad\right|_{e_{j}(t) \in R}$ and $a_{2}=\left.\min \left\{\partial \phi\left(u_{j}(t)\right) / \partial u_{j}\right\} \quad\right|_{u_{j}(t) \in R}$ that are gained by applying the mean-value theorem two times. As seen from (18), we can easily draw a conclusion $\dot{s}_{j}(t) \leq 0$ provided that

$$
\frac{\gamma_{2} a_{2}}{\zeta}-\frac{\gamma_{2}^{2} a_{0}^{2}}{4 \gamma_{1} a_{1}} \geq 0 \text { and } \zeta>0 \text {, i.e, } 0<\zeta \leq \frac{4 \gamma_{1} a_{1} a_{2}}{\gamma_{2} a_{0}^{2}} \text {. }
$$

Based on Lyapunov stability theory, we know that the $j$ th subsystem (10) is globally stable. Since the second-order nonlinear system (9) is consist of $n$ subsystems of (10), we conclude that the second-order nonlinear system (9) is globally stable as long as $\Phi(\cdot)$ is a monotonic increasing odd function. This completes the proof.

Theorem 2: The second-order nonlinear system (9) is capable of converging to the equilibrium point within finite time, and its convergence upper bound $t_{\mathrm{f}}$ is

$$
t_{\mathrm{f}}<\frac{\gamma_{1}+\gamma_{2}}{\gamma_{1} \gamma_{2}(1-p)} \max \left\{\left|e^{-}(0)\right|^{1-p},\left|e^{+}(0)\right|^{1-p}\right\},
$$

provided that $\phi(e)=\left(|e|^{p}+|e|^{1 / p}\right) \operatorname{sgn}(e)$ with $0<p<1$, where $\operatorname{sgn}(\cdot)$ denotes the sign function, and the initial errors $e^{+}(0)=\max \{\mathbf{e}(t)\}$ and $e^{-}(0)=\min \{\mathbf{e}(t)\}$.

Proof: For the $j$ th subsystem of (9), via introducing $u_{j}(t)=e_{j}(t)+\gamma_{1} \int_{0}^{t} \phi\left(e_{j}(\tau)\right) \mathrm{d} \tau$, we are capable of gaining $\dot{u}_{j}(t)=-\gamma_{2} \phi\left(u_{j}(t)\right)$. Especially, when $t=0$, we can obtain $u_{j}(0)=e_{j}(0)$. Besides, the Lyapunov function candidate $s_{j}=u_{j}^{2}(t)$ is selected to compute finite convergence time of nonlinear dynamic system $\dot{u}_{j}(t)=-\gamma_{2} \phi\left(u_{j}(t)\right)$ [44]-[46]. Its time derivative is computed as below:

$$
\begin{aligned}
\dot{s}_{j} & =2 u_{j}(t) \dot{u}_{j}(t) \\
& =-2 \gamma_{2} u_{j}(t) \phi\left(u_{j}(t)\right) \\
& =-2 \gamma_{2}\left(\left|u_{j}(t)\right|^{p+1}+\left|u_{j}(t)\right|^{\frac{1}{p}+1}\right) \\
& \leq-2 \gamma_{2}\left|u_{j}(t)\right|^{p+1} \\
& =-2 \gamma_{2} s_{j}^{\frac{p+1}{2}},
\end{aligned}
$$

where $\phi\left(u_{j}\right)=\left(\left|u_{j}\right|^{p}+\left|u_{j}\right|^{1 / p}\right) \operatorname{sgn}\left(u_{j}\right)$. Then, solving the inequality $\dot{s}_{j} \leqslant-2 \gamma_{2} s_{j}{ }^{\frac{p+1}{2}}$ with $s_{j}(0)=\left|u_{j}(0)\right|^{2}=\left|e_{j}(0)\right|^{2}$, one can obtain:

$$
s_{j}^{\frac{1-p}{2}}(t) \begin{cases}\leq\left|u_{j}(0)\right|^{1-p}-\gamma_{2} t(1-p), & \text { if } t \leq \frac{\left|u_{j}(0)\right|^{1-p}}{\gamma_{2}(1-p)}, \\ =0, & \text { if } t>\frac{\left|u_{j}(0)\right|^{1-p}}{\gamma_{2}(1-p)}\end{cases}
$$


which shows that $s_{j}$ converges to zero when $t>$ $\left|u_{j}(0)\right|^{1-p} / \gamma_{2}(1-p)$. Owing to $s_{j}=u_{j}^{2}(t)$ and $u_{j}(0)=$ $e_{j}(0)$, it can also be concluded that $u_{j}(t)$ converges to zero after $t>\left|e_{j}(0)\right|^{1-p} / \gamma_{2}(1-p)$.

Since all elements in $\mathbf{u}(t)$ have the same dynamics $\dot{u}_{j}(t)=-\gamma_{2} \phi\left(u_{j}(t)\right), \mathbf{u}(t)$ converges to zero when $t>$ $\max \left\{\left|e^{-}(0)\right|^{1-p},\left|e^{+}(0)\right|^{1-p}\right\} / \gamma_{2}(1-p)$, where $e^{+}(0)=$ $\max \{\mathbf{e}(t)\}$ and $e^{-(0)}=\min \{\mathbf{e}(t)\}$. Therefore, the upper bound $t_{1}$ for $\mathbf{u}(t)$ is calculated as

$$
t_{1}<\frac{1}{\gamma_{2}(1-p)} \max \left\{\left|e^{-}(0)\right|^{1-p},\left|e^{+}(0)\right|^{1-p}\right\} .
$$

The convergence upper bound for $\mathbf{u}(t)$ is thus completed.

From the above discussion, when $t>t_{1}, \mathbf{u}(t)$ converges to the equilibrium point, and thus $\dot{\mathbf{u}}(t)=0$. Based on (12), when $t>t_{1}$, we have

$$
\dot{e}_{j}(t)+\gamma_{1} \phi\left(e_{j}(t)\right)=0,
$$

which is exactly the same form of $\dot{u}_{j}(t)+\gamma_{2} \phi\left(u_{j}(t)\right)=0$. Considering the different parameters of these two dynamic systems, we are able to compute the convergence upper bound $t_{2}$ as below:

$$
t_{2}<\frac{1}{\gamma_{1}(1-p)} \max \left\{\left|e^{-}(0)\right|^{1-p},\left|e^{+}(0)\right|^{1-p}\right\},
$$

where $e^{+}(0)$ and $e^{-}(0)$ are defined as before.

All in all, by generalizing the above two conclusions, one can conclude that the second-order nonlinear system (9) is capable of converging to the equilibrium point in a limited time, and its convergence upper bound $t_{\mathrm{f}}$ is

$$
t_{\mathrm{f}}<t_{1}+t_{2}=\frac{\gamma_{1}+\gamma_{2}}{\gamma_{1} \gamma_{2}(1-p)} \max \left\{\left|e^{-}(0)\right|^{1-p},\left|e^{+}(0)\right|^{1-p}\right\} .
$$

This completes the proof.

In order to study the robustness property of the second-order nonlinear system (9) when external disturbances are injected into this system, we consider an unknown additive constant noise $v$. Thus, the noise-disturbed second-order nonlinear dynamic system can be described as

$$
\dot{\mathbf{e}}(t)=-\gamma_{1} \Phi(\mathbf{e}(t))-\gamma_{2} \Phi\left(\mathbf{e}(t)+\gamma_{1} \int_{0}^{t} \Phi(\mathbf{e}(\tau)) \mathrm{d} \tau\right)+v
$$

where $v$ represents an unknown additive constant noise. Next, let us prove the inherent noise tolerant property of the above the noise-disturbed nonlinear dynamic system (21).

Theorem 3: The noise-disturbed second-order nonlinear dynamic system (21) is capable of globally converging to zero under additive constant noise $v$.

Proof: Let us consider the $j$ th subsystem of (21), which is described as:

$$
\dot{e}_{j}(t)=-\gamma_{1} \phi\left(e_{j}(t)\right)-\gamma_{2} \phi\left(e_{j}(t)+\gamma_{1} \int_{0}^{t} \phi\left(e_{j}(\tau)\right) \mathrm{d} \tau\right)+v .
$$

As the same as Theorem 1, we also introduce a new variable $u_{j}(t)$, which is defined as the same with (11). Its time derivative is thus gained as $\dot{u}_{j}(t)=\dot{e}_{j}(t)+\gamma_{1} \phi\left(e_{j}(t)\right)$. Then, substituting the expressions of $u_{j}(t)$ and $\dot{u}_{j}(t)$ into (22), we have the fact:

$$
\dot{u}_{j}(t)=-\gamma_{2} \phi\left(u_{j}(t)\right)+v .
$$

According to the above results, we are capable of defining the following Lyapunov function for the $j$ th subsystem (22):

$$
s_{j}(t)=\left(\gamma_{2} \phi\left(u_{j}(t)\right)-v\right)^{2} / 2 .
$$

Its time derivative $\dot{s}_{j}(t)$ is gained as below:

$$
\begin{aligned}
\frac{\mathrm{d} s_{j}(t)}{\mathrm{d} t} & =\left(\gamma_{2} \phi\left(u_{j}(t)\right)-v\right) \gamma_{2} \frac{\partial \phi\left(u_{j}(t)\right)}{\partial u_{j}} \dot{u}_{j}(t) \\
& =-\gamma_{2} \frac{\partial \phi\left(u_{j}(t)\right)}{\partial u_{j}}\left(\gamma_{2} \phi\left(u_{j}(t)\right)-v\right)^{2} .
\end{aligned}
$$

Since $\phi(\cdot)$ is a monotonic increasing odd activation function, we have $\partial \phi\left(u_{j}(t)\right) / \partial u_{j}>0$. Therefore, we can obtain $\dot{s}_{j}(t) \leq 0$, and $\lim _{t \rightarrow \infty} s_{j}(t)=0$. At this time, $\lim _{t \rightarrow \infty} \gamma_{2} \phi\left(u_{j}(t)\right)-v=0$ and $\lim _{t \rightarrow \infty} u_{j}(t)=\phi_{2}^{-1}\left(v / \gamma_{2}\right)$. Thus, we have $\lim _{t \rightarrow \infty} \dot{u}_{j}(t)=-\gamma_{2} \phi\left(u_{j}(t)\right)+v=0$.

On the other hand, due to $\dot{u}_{j}(t)=\dot{e}_{j}(t)+\gamma_{1} \phi\left(e_{j}(t)\right)$ and $\lim _{t \rightarrow \infty} \dot{u}_{j}(t)=0$, and basis on Lasalle's invariant set principle [47]-[49], it can be concluded that $\dot{u}_{j}(t)=$ $\dot{e}_{j}(t)+\gamma_{1} \phi\left(e_{j}(t)\right)$ reduces to

$$
\dot{e}_{j}(t)+\gamma_{1} \phi\left(e_{j}(t)\right)=0,
$$

which is the aforementioned nonlinear system. In addition, it has been proved that this nonlinear dynamic system is capable of converging to its equilibrium point exponentially.

According to the above analyses, we conclude that, under unknown additive constant noise $v$, the noise-disturbed second-order nonlinear dynamic system (21) is capable of globally converging to zero. This completes the proof.

\section{B. LTRNN Model}

Based on the above proposed second-order nonlinear dynamic formula (9), a limited-time robust neural network (LTRNN) has been established and analyzed for time-dependent nonlinear minimization (1) and its equivalent nonlinear equation system (2). The detailed design process and theoretical analysis are presented as follows.

At first, we are capable of defining the following monitor error function similarly:

$$
\mathbf{e}(t)=\left[\frac{\partial g(\mathbf{x}(t), t)}{\partial x_{1}}, \frac{\partial g(\mathbf{x}(t), t)}{\partial x_{2}}, \cdots, \frac{\partial g(\mathbf{x}(t), t)}{\partial x_{n}}\right]^{\mathrm{T}} .
$$

Then, according to error function (26), the proposed secondorder nonlinear dynamic formula is adopted to establish the LTRNN model. For maintaining the coherence of reading, such a second-order nonlinear dynamic formula is presented again as below:

$$
\dot{\mathbf{e}}(t)+\gamma_{1} \Phi(\mathbf{e}(t))+\gamma_{2} \Phi\left(\mathbf{e}(t)+\gamma_{1} \int_{0}^{t} \Phi(\mathbf{e}(\tau)) \mathrm{d} \tau\right)=\mathbf{0} .
$$

Substituting (26) into the above second-order nonlinear dynamic formula and considering $\mathbf{e}(t)=\partial g(\mathbf{x}(t), t) / \partial \mathbf{x}(t)=$ $\mathbf{z}(\mathbf{x}(t), t)$, we are capable of gaining the LTRNN model for 


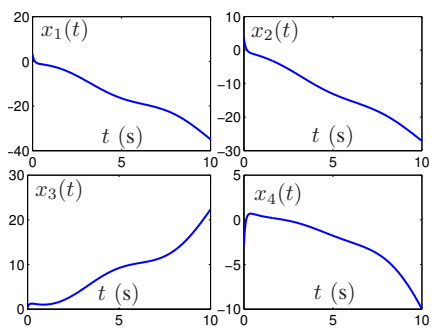

(a)

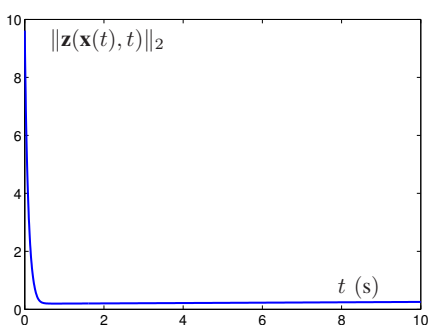

(b)
Fig. 1. Computing nonlinear minimization problem by $\mathrm{ZNN}$ model (6) using the sign-bi-power activation function with $\varpi=5$ in front of constant noise $v=0.5$. (a) Neural output $\mathbf{x}(t)$. (b) Residual error $\|\mathbf{z}(\mathbf{x}(t), t)\|_{2}$.

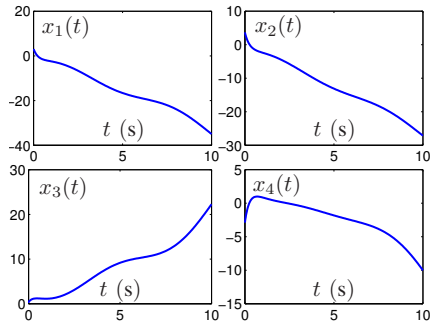

(a)

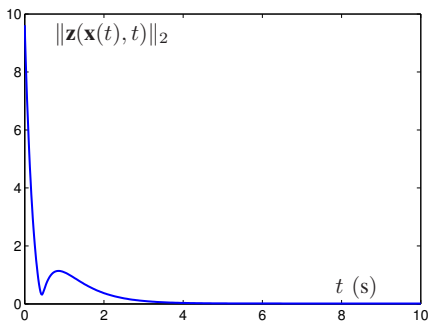

(b)
Fig. 2. Computing nonlinear minimization problem by RZNN model (8) with $\gamma_{1}=\gamma_{2}=5$ in front of constant noise $v=0.5$. (a) Neural output $\mathbf{x}(t)$. (b) Residual error $\|\mathbf{z}(\mathbf{x}(t), t)\|_{2}$.

solving time-dependent nonlinear minimization (1) and its equivalent nonlinear equation system (2) as below:

$$
\mathbf{Q} \dot{\mathbf{x}}=-\gamma_{1} \Phi(\mathbf{z})-\gamma_{2} \Phi\left(\mathbf{z}+\gamma_{1} \int_{0}^{t} \Phi(\mathbf{z}) \mathrm{d} \tau\right)-\frac{\partial \mathbf{z}}{\partial t}
$$

where independent variable $t$ is omitted for presentation convenience; and coefficient matrix $\mathbf{Q}$ and vector $\frac{\partial \mathbf{z}}{\partial t}$ are defined as the same ones of RZNN model (8).

If external disturbances are injected in LTRNN model (27), the noise-tolerant LTRNN model is directly given below via considering the noise-disturbed second-order nonlinear dynamic system (21):

$$
\mathbf{Q} \dot{\mathbf{x}}=-\gamma_{1} \Phi(\mathbf{z})-\gamma_{2} \Phi\left(\mathbf{z}+\gamma_{1} \int_{0}^{t} \Phi(\mathbf{z}) \mathrm{d} \tau\right)-\frac{\partial \mathbf{z}}{\partial t}+v .
$$

After proposing the above LTRNN models, we proceed to prove the superior finite-convergence and noise-tolerant properties via the following theorems.

Theorem 4: The neural output $\mathbf{x}(t)$ of LTRNN model (27) is capable of converging to the optimal solution $\mathbf{x}^{*}(t)$ of timedependent nonlinear minimization (1).

Proof: As observed in the design process of LTRNN model (27), we can conclude that LTRNN model (27) is an equivalent extended form of the second-order nonlinear formula (9) via defining $\mathbf{e}(t)=\frac{\partial g(\mathbf{x}(t), t)}{\partial \mathbf{x}(t)}=\mathbf{z}(\mathbf{x}(t), t)$. Then, according to Theorem 1, it follows that LTRNN model (27) is globally stable. Therefore, neural output $\mathbf{x}(t)$ of the LTRNN model (27) globally converges to the optimal solution $\mathbf{x}^{*}(t)$ of timedependent nonlinear minimization (1). This completes proof.

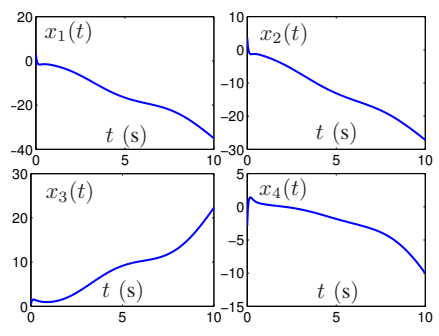

(a)

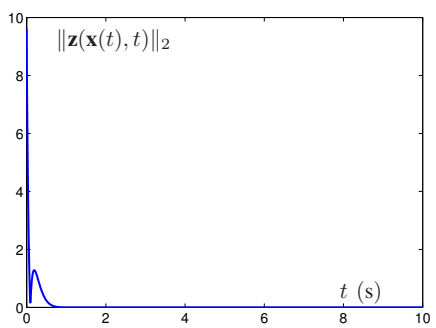

(b)
Fig. 3. Computing nonlinear minimization problem by LTRNN model (27) using the sign-bi-power activation function with $\gamma_{1}=\gamma_{2}=5$ in front of constant noise $v=0.5$. (a) Neural output $\mathbf{x}(t)$. (b) Residual error $\|\mathbf{z}(\mathbf{x}(t), t)\|_{2}$.

Theorem 5: The neural output $\mathbf{x}(t)$ of LTRNN model (27) is capable of converging to the optimal solution $\mathbf{x}^{*}(t)$ of timedependent nonlinear minimization (1) within finite time, with the convergence upper bound $t_{\mathrm{f}}$ being

$$
t_{\mathrm{f}}<\frac{\gamma_{1}+\gamma_{2}}{\gamma_{1} \gamma_{2}(1-p)} \max \left\{\left|e^{-}(0)\right|^{1-p},\left|e^{+}(0)\right|^{1-p}\right\},
$$

as long as $\phi(e)=\left(|e|^{p}+|e|^{1 / p}\right) \operatorname{sgn}(e)$ with $0<p<$ 1 , where $\operatorname{sgn}(\cdot)$ denotes the sign function, and $e^{+}(0)=$ $\max \{\mathbf{e}(t)\}$ and $e^{-}(0)=\min \{\mathbf{e}(t)\}$.

Proof: We can complete the proof in a similar way according to previous theorems.

Theorem 6: The neural output $\mathbf{x}(t)$ of the noise tolerant LTRNN model (28) is capable of converging to the optimal solution $\mathbf{x}^{*}(t)$ of time-dependent nonlinear minimization (1) even in the presence of unknown additive constant noises.

Proof: We can complete the proof in a similar way according to previous theorems.

\section{NUMERICAL VERIFICATIONS}

To demonstrate the superior property of LTRNN model (27) for time-dependent nonlinear minimization (2), ZNN model (6) and RZNN model (8) are also applied to solve a timedependent nonlinear minimization problem under various different noises. Note that ZNN (6) and LTRNN (27) are activated by the sign-bi-power function $\phi(e)=\left(|e|^{p}+|e|^{1 / p}\right) \operatorname{sgn}(e)$ with $p=0.8$. Now, we consider the following solvable nonlinear minimization example:

$$
\min _{\mathbf{x}(t) \in R^{4}} g(\mathbf{x}(t), t), \quad \forall t \in[0, \infty)
$$

where $\mathbf{x}(t)=\left[x_{1}(t), x_{2}(t), x_{3}(t), x_{4}(t)\right]^{\mathrm{T}}$ and the expression of $g(\mathbf{x}(t), t)$ is defined as

$$
\begin{aligned}
g= & \left(x_{1}+t\right)^{2}+\left(x_{2}+t\right)^{2}+\left(x_{3}-\exp (-t)\right)^{2} \\
& +\left(x_{4}-\exp (-t)\right)^{2}+\left(x_{1}+\sin (t)\right) x_{3} \\
& -\left(x_{1}+\ln (0.1 t+1)\right)\left(x_{2}+\sin (t)\right)+0.1(t-1) x_{3} x_{4}
\end{aligned}
$$

with independent variable $t$ deleted for presentation convenience. Furthermore, $\mathbf{z}(\mathbf{x}(t), t)$ can be obtained as

$$
\mathbf{z}=\left\{\begin{array}{l}
2\left(x_{1}+t\right)+x_{3}-\left(x_{2}+\sin (t)\right)=0 \\
2\left(x_{2}+t\right)-\left(x_{1}+\ln (0.1 t+1)\right)=0 \\
2\left(x_{3}-\exp (-t)\right)+\left(x_{1}+\sin (t)\right)+0.1(t-1) x_{4}=0 \\
2\left(x_{4}-\exp (-t)\right)+0.1(t-1) x_{3}=0
\end{array}\right.
$$




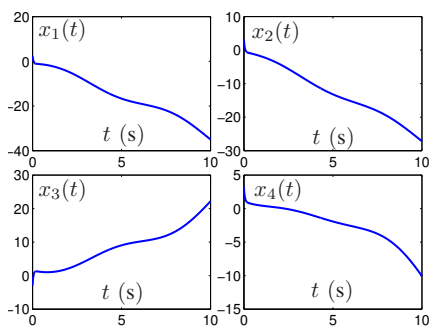

(a)

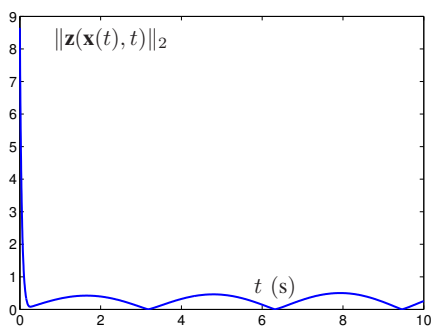

(b)
Fig. 4. Computing nonlinear minimization problem by ZNN model (6) using the sign-bi-power activation function with $\varpi=10$ under constant noise $v=2 \sin (t)$. (a) Neural output $\mathbf{x}(t)$. (b) Residual error $\|\mathbf{z}(\mathbf{x}(t), t)\|_{2}$.

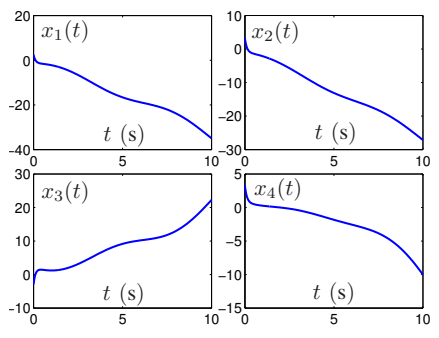

(a)

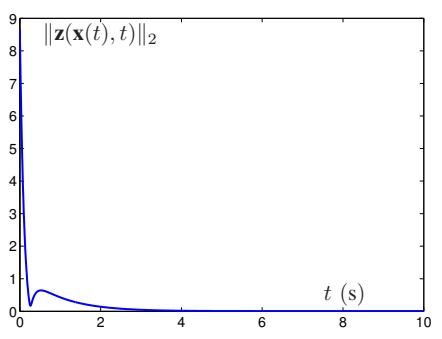

(b)
Fig. 5. Computing nonlinear minimization problem by RZNN model (8) with $\gamma_{1}=\gamma_{2}=10$ under dynamic noise $v=2 \sin (t)$. (a) Neural output $\mathbf{x}(t)$. (b) Residual error $\|\mathbf{z}(\mathbf{x}(t), t)\|_{2}$.

In this example, we consider different situations according to the types of external disturbances. In general, constant and dynamic noises are two kinds of major representatives of external disturbances. Therefore, in the following simulations, we mainly consider the constant and dynamic noises as external disturbances.

1) Constant Noise: First, the constant noise $v=0.5$ is taken into account. From a starting point located in $\mathbf{x}(0) \in$ $[-4,4]^{4}$, such three neural-network models are explored to address the above nonlinear minimization problem under the same conditions. Computer simulative results are comparatively displayed in Figs. 1-3. Figure 1 displays the computing results generated by ZNN (6) under design parameter $\varpi=5$. Because ZNN model (6) is activated by the sign-bi-power function, the residual error decreases quickly at first. However, $\|\mathbf{z}(\mathbf{x}(t), t)\|_{2}$ does not converge to 0 finally. That is to say, ZNN model (6) cannot suppress external noises so that it generates a relatively large error. Figure 2 shows the computing results generated by RZNN model (8) with design parameters $\gamma_{1}=\gamma_{2}=5$. As seen from this figure, the residual error $\|\mathbf{z}(\mathbf{x}(t), t)\|_{2}$ can converge to 0 but it need about $5 \mathrm{~s}$. The convergence time is relatively longer. At last, under the same conditions, Figure 3 shows the computing results generated by LTRNN model (27) with design parameters $\gamma_{1}=\gamma_{2}=5$. It follows from this figure that $\|\mathbf{z}(\mathbf{x}(t), t)\|_{2}$ can decrease to 0 quickly within limited time $0.8 \mathrm{~s}$. The convergence speed of residual error $\|\mathbf{z}(\mathbf{x}(t), t)\|_{2}$ solved by LTRNN model (27) is about 6 times faster than that by RZNN model (8). The results show that LTRNN model (27) is a best model for solving timedependent nonlinear minimization (2) under constant noise, compared to ZNN model (6) and RZNN model (8).

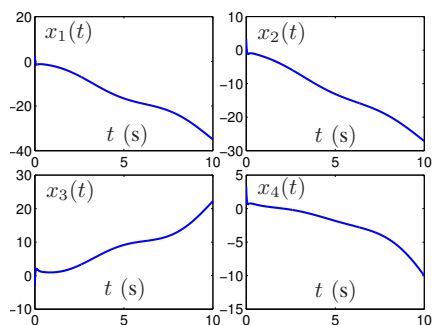

(a)

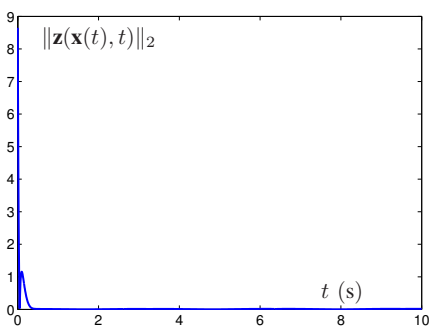

(b)
Fig. 6. Computing nonlinear minimization problem by LTRNN model (27) using the sign-bi-power activation function with $\gamma_{1}=\gamma_{2}=10$ in front of dynamic noise $v=2 \sin (t)$. (a) Neural output $\mathbf{x}(t)$. (b) Residual error $\|\mathbf{z}(\mathbf{x}(t), t)\|_{2}$.

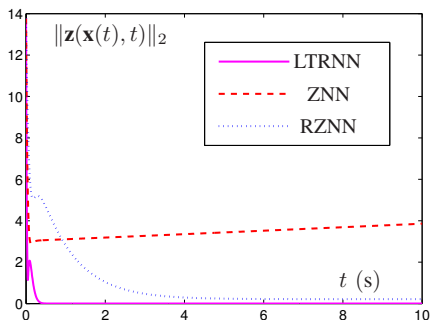

(a)

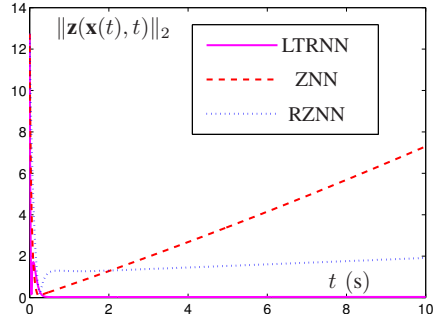

(b)
Fig. 7. Residual error $\|\mathbf{z}(\mathbf{x}(t), t)\|_{2}$ generated by LTRNN model (8), ZNN model (6) and RZNN model (8) with $\varpi=\gamma_{1}=\gamma_{2}=10$ in front of different types of noises. (a) Bounded additive noise $v=15$. (b) Linearly increasing noise $v=3 t$.

2) Dynamic Noise: In this part, a more general situation is considered: dynamic noise, which exists more frequently in practical engineering fields. Without losing generality, such a dynamic noise is set as $v=2 \sin (t)$. Then, we apply $\mathrm{ZNN}(6)$, RZNN (8) and LTRNN (27) to compute the above example under dynamic noise $v=2 \sin (t)$. With design parameters $\varpi=\gamma_{1}=\gamma_{2}=10$, and from a starting point located in $\mathbf{x}(0) \in[-4,4]^{4}$, simulative results are comparatively generated in Figs. 4-6, from which we are able to draw a conclusion that the residual error generated by $\mathrm{ZNN}$ (6) is always changing with the direction of the dynamic noise $v=2 \sin (t)$; the residual error generated by RZNN (8) can decrease to 0 but its convergence speed is slow; and the residual error generated by LTRNN model (27) is capable of decreasing to zero with finite time $0.5 \mathrm{~s}$ and the convergence time is the shortest. Although the additive noise is dynamic, LTRNN model (27) is still capable of suppressing the external disturbance. In addition, the convergence speed still achieves finite time. These facts further demonstrate the advantage of LTRNN model (27) for solving time-dependent nonlinear minimization problems.

We conduct further simulations by using such three models with other conditions unchanged under different types of noises. The bounded additive noise is considered firstly, which is set as $v=15$. With the other conditions unchanged, the convergence behavior of residual error $\|\mathbf{z}(\mathbf{x}(t), t)\|_{2}$ produced by LTRNN (27), ZNN (6) and RZNN (8) is shown in Fig. 7(a). When disturbed by the bounded noise, LTRNN model (27) can still achieve noise suppression and limited-time convergence, while ZNN model (6) and RZNN model (8) cannot converge to 0 within limited time. Besides, we further investigate the 
linearly increasing noise, which is set as $v=3 t$. With other conditions unchanged, the corresponding convergence behavior of residual error $\|\mathbf{z}(\mathbf{x}(t), t)\|_{2}$ is shown in Fig. 7(b), which demonstrates that LTRNN model (27) is still effective, while the other models completely lose efficacy.

In brief, we reach a decision that LTRNN model (27) is the best model for solving time-dependent nonlinear minimization problem even under various external disturbances.

\section{CONCLUSION}

Based on the second-order nonlinear design formula, the LTRNN model has been established according to the method of ZNN for time-dependent nonlinear minimization. Rigorous theoretical analyses have been given to simultaneously achieve limited-time convergence and inherently noise suppression by LTRNN. In order to highlight the outstanding advantage of LTRNN, ZNN and its improved model have been applied to time-dependant nonlinear minimization solving. Comparative numerical results have further validated the efficacy and advantage of LTRNN for nonlinear minimization. This work is for the first time to solve time-dependent nonlinear minimization in noisy environments by devising LTRNN with limited-time convergence and noise tolerance simultaneously, making a progress in theory. The future work is to apply the LTRNN model to some practical applications.

\section{REFERENCES}

[1] Y. Zhang, H. Gong, M. Yang, J. Li, and X. Yang, "Stepsize range and optimal value for Taylor-Zhang discretization formula applied to zeroing neurodynamics illustrated via future equality-constrained quadratic programming," IEEE Trans. Neural Netw. Learning Syst., vol. 30, no. 3, pp. 959-966, 2019.

[2] H. Wang, P. X. Liu, S. Li, and D. Wang, "Adaptive neural outputfeedback control for a class of nonlower triangular nonlinear systems with unmodeled dynamics," IEEE Trans. Neural Netw. Learning Syst., vol. 29, no. 8, pp. 3658-3668, 2017.

[3] L. Xiao, Z. Zhang, and S. Li, "Solving time-varying system of nonlinear equations by finite-time recurrent neural networks with application to motion tracking of robot manipulators," IEEE Trans. Syst., Man, Cybern., Syst., In press, 2019.

[4] Z. Zhang, L. Zheng, J. Weng, Y. Mao, W. Lu, and L. Xiao, "A new varying-parameter recurrent neural-network for online solution of timevarying Sylvester equation," IEEE Trans. Cybern., vol. 48, no. 11, pp. 3135-3148, 2018.

[5] X. Xu, Z. Huang, L. Zuo, and H. He, "Manifold-based reinforcement learning via locally linear reconstruction," IEEE Trans. Neural Netw. Learning Syst., vol. 28, no. 4, pp. 934-947, 2017.

[6] J. Xu, B. Tang, H. He, H. Man, "Semisupervised feature selection based on relevance and redundancy criteria," IEEE Trans. Neural Netw. Learning Syst., vol. 28, no. 9, pp. 1974-1984, 2017.

[7] D. Chen and Y. Zhang, "Robust zeroing neural-dynamics and its timevarying disturbances suppression model applied to mobile robot manipulators," IEEE Trans. Neural Netw. Learning Syst., vol. 29, no. 9, pp. 4385-4397, 2018

[8] Y. Zhang, and S. Li, "Predictive suboptimal consensus of multiagent systems with nonlinear dynamics," IEEE Trans. Syst., Man, Cybern., Syst., vol. 47, no. 7, pp. 1701-1711, Jul. 2017.

[9] W. Li, B. Liao, L. Xiao, and R. Lu, "A recurrent neural network with predefined-time convergence and improved noise tolerance for dynamic matrix square root finding," Neurocomputing, vol. 337,pp. 262-273, 2019.

[10] P. Ochs, A. Dosovitskiy, T. Brox, and T. Pock, "On iteratively reweighted algorithms for nonsmooth nonconvex optimization in computer vision," SIAM J. Imag. Sci., vol.8, no. 1, pp. 331-372, 2015

[11] I. Necoara and A. Patrascu, "A random coordinate descent algorithm for optimization problems with composite objective function and linear coupled constraints," Comput. Optim. Appl., vol. 57, no. 2, pp. 307-337, 2014.
[12] S. Abbasbandy, "Improving Newton-Raphson method for nonlinear equations by modified Adomian decomposition method," Appl. Math. Comput., vol. 145, pp. 887-893, 2003.

[13] S. Abbasbandy, Y. Tan, and S. J. Liao, "Newton-homotopy analysis method for nonlinear equations," Appl. Math. Comput., vol. 188, pp. 1794-1800, 2007.

[14] C. Chun, and M. Y. Lee, "A new optimal eighth-order family of iterative methods for the solution of nonlinear equations," Appl. Math. Comput., vol. 223, pp. 506-519, 2013.

[15] D. Bertsekas, Constrained Optimization and Lagrange Multiplier Methods, Academic press. 2014.

[16] D. Guo, Y. Zhang, "Neural dynamics and Newton-Raphson iteration for nonlinear optimization," ASME J. Comput. Nonlinear Dyn. vol. 9, no. 2, p. 021016, 2014.

[17] J. H. Mathews and K. D. Fink, Numerical Methods Using MATLAB. Englewood Cliffs, NJ, USA: Prentice-Hall, 2004.

[18] H. Dong, Z. Wang, S. X., Ding, and H. Gao, "Finite-horizon estimation of randomly occurring faults for a class of nonlinear time-varying systems," Automatica, vol. 50, no. 12, pp. 3182-3189, 2014.

[19] L. Xiao, Y. Zhang, Z. Hu, and J. Dai, "Performance benefits of robust nonlinear zeroing neural network for finding accurate solution of Lyapunov equation in presence of various noises," IEEE Trans. Ind. Informat., vol. 15, no. 9, pp. 5161-5171, 2019.

[20] L. Xiao, "Design and analysis of robust nonlinear neural dynamics for solving dynamic nonlinear equation within finite time," Nonlinear Dyn., vol. 96 , no. 4, pp. 2437-2447, 2019.

[21] L. Dong, X. Zhong, C. Sun, H. He, "Event-triggered adaptive dynamic programming for continuous-time systems with control constraints," IEEE Trans. Neural Netw. Learning Syst., vol. 28, no. 8, pp. 1941-1952, 2017.

[22] Z. Zhang, Z. Yan, and T. Fu, "Varying-parameter RNN activated by finite-time functions for solving joint-drift problems of redundant robot manipulators," IEEE Trans. Ind. Inform., vol. 14, no. 12, pp. 5359-5367, 2018.

[23] B. Liao, Y. Zhang, and L. Jin, "Taylor $O\left(h^{3}\right)$ discretization of ZNN models for dynamic equality-constrained quadratic programming with application to manipulators," IEEE Trans. Neural Netw. Learning Syst., vol. 27, no. 2, pp. 225-237, 2016.

[24] S. Li, B. Liu, and Y. Li, "Selective positive-negative feedback produces the winner-take-all competition in recurrent neural networks," IEEE Trans. Neural Netw. Learning Syst., vol. 24, no. 2, pp. 301-309, Feb. 2013.

[25] Z. Tan, W. Li, L. Xiao, and Y. Hu, "New varying-parameter ZNN models with finite-time convergence and noise suppression for timevarying matrix Moore-Penrose inversion," IEEE Trans. Neural Netw. Learning Syst., In press, 2019.

[26] Y. Zhang, C. Yi, D. Guo, and J. Zheng, "Comparison on Zhang neural dynamics and gradient-based neural dynamics for online solution of nonlinear time-varying equation," Neural Comput. Appl., vol. 20, pp. 1-7, 2011.

[27] K. Chen, "Recurrent implicit dynamics for online matrix inversion," Appl. Math. Comput., vol. 219, no. 20, pp. 10218-10224, Jun. 2013.

[28] L. Xiao, S. Li, F. Lin, Z. Tan, and A. Khan, "Zeroing neural dynamics for control design: comprehensive analysis on stability, robustness, and convergence speed," IEEE Trans. Ind. Inform., vol. 15, no. 5, pp. 2605 2616, 2019.

[29] C. Yi, Y. Chen, and Z. Lu, "Improved gradient-based neural networks for online solution of Lyapunov matrix equation," Inf. Process. Lett., vol. 111, no. 16, pp. 780-786, Aug. 2011.

[30] Y. Chen, C. Yi, and D. Qiao, "Improved neural solution for the Lyapunov matrix equation based on gradient search," Inf. Process. Lett., vol. 13, no. 22-24, pp. 876-881, Nov. 2013.

[31] Y. Zhang, D. Jiang, and J.Wang, "A recurrent neural network for solving Sylvester equation with time-varying coefficients," IEEE Trans. Neural Netw., vol. 13, no. 5, pp. 1053-1063, 2002.

[32] Y. Zhang and S. S. Ge, "Design and analysis of a general recurrent neural network model for time-varying matrix inversion," IEEE Trans. Neural Netw., vol. 16, no. 6, pp. 1477-1490, 2005.

[33] L. Xiao, Y. Zhang, et al., "A new noise-tolerant and predefined-time ZNN model for time-dependent matrix inversion," Neural Netw., vol. 117, pp. 124-134, 2019.

[34] S. Li, Y. Li, and Z. Wang, "A class of finite-time dual neural networks for solving quadratic programming problems and its k-winners-take-all application," Neural Netw, vol. 39, pp. 27-39, 2013.

[35] L. Xiao, and B. Liao, "A convergence-accelerated Zhang neural network and its solution application to Lyapunov equation," Neurocomputing, vol. 193, pp. 213-218, 2016 
[36] L. Xiao, B. Liao, S. Li, and K. Chen, "Nonlinear recurrent neural networks for finite-time solution of general time-varying linear matrix equations," Neural Netw., vol. 98, pp. 102-113, 2018.

[37] L. Xiao, "A finite-time convergent Zhang neural network and its application to real-time matrix square root finding," Neur Comput Appl., vol. 31, no. 2, pp. 793-800, 2019.

[38] L. Xiao, B. Liao, S. Li, Z. Zhang, L. Ding, and L. Jin, "Design and analysis of FTZNN applied to the real-time solution of a nonstationary Lyapunov equation and tracking control of a wheeled mobile manipulator," IEEE Trans. Ind. Inform., vol. 14, no.1, pp. 98-105, 2018.

[39] L. Jin and Y. Zhang, "Continuous and discrete Zhang dynamics for realtime varying nonlinear optimization," Numer. Algor., vol. 73, pp. 115140,2016

[40] L. Jin and Y. Zhang, "Discrete-time Zhang neural network for online time-varying nonlinear optimization with application to manipulator motion generation," IEEE Trans. Neural Netw. Learning Syst., vol. 26, no. 7, pp. 1525-1531, 2015

[41] L. Jin, Y. Zhang, and S. Li, "Integration-enhanced Zhang neural network for real-time-varying matrix inversion in the presence of various kinds of noises," IEEE Trans. Neural Netw. Learning Syst., vol. 27, no. 12, pp. 2615-2627, 2016

[42] L. Xiao, S. Li, K. Li, L. Jin, and B. Liao, "Co-design of finite-time convergence and noise suppression: A unified neural model for time varying linear equations with robotic applications," IEEE Trans. Syst., Man, Cybern., Syst., In press, 2018.

[43] W. Li, L. Xiao, and B. Liao "A finite-time convergent and noise-rejection recurrent neural network and its discretization for dynamic nonlinear equations solving," IEEE Trans. Cybern., In press, 2019.

[44] Y. Zhang, Y. Ding, B. Qiu, Y. Zhang, and X. Li, "Signum-function array activated ZNN with easier circuit implementation and finite-time convergence for linear systems solving," Inf. Process. Lett., vol. 124, pp. 30-34, 2017.

[45] S. Li, S. Chen, and B. Liu, "Accelerating a recurrent neural network to finite-time convergence for solving time-varying Sylvester equation by using a sign-bi-power activation function," Neural Process. Lett., vol. 37, pp. 189-205, 2013.

[46] L. Xiao, and R. Lu, "Finite-time solution to nonlinear equation using recurrent neural dynamics with a specially-constructed activation function," Neurocomputing, vol. 151, pp. 246-251, 2015.

[47] L. Jin, S. Li, L. Xiao, R. Lu, and B. Liao, "Cooperative motion generation in a distributed network of redundant robot manipulators with noises," IEEE Trans. Syst. Man Cybern. Syst., vol. 99, no. 10, pp. 17151724, 2018.

[48] Z. Tan, L. Xiao, S. Chen, and X. Lv, "Noise-tolerant and finite-time convergent ZNN models for dynamic matrix Moore-Penrose inversion," IEEE Trans. Ind. Inform., In press, 2019.

[49] L. Xiao, K. Li, and M. Duan, "Computing time-varying quadratic optimization with finite-time convergence and noise tolerance: A unified framework for zeroing neural network," IEEE Trans. Neural Netw. Learning Syst., In press, 2019. 